\title{
A Convenient and Efficient Method for the Synthesis of a Novel Series of $N$-Butyl-1,4-oxazino[2',3':4,5]pyrano[3,2-c] quinolinones
}

\author{
Hany M. Hassanin and Mohamed M. Hassan* \\ Department of Chemistry, Faculty of Education, Ain Shams University, Roxy, 11711 Cairo, Egypt
}

\begin{abstract}
We design and describe here a simple route for the synthesis of a new multi heterocycle fused 1,4-oxazinopyranoquinolinones compatible with many functional groups. This method proceeds through various cyclic condensation reactions of (3-amino or 3-nitropyrano)[3,2-c] quinoline2,5(6H)-dione precursors with different 1,2-bifunctional electrophiles. The synthesized compounds are confirmed by infrared (IR), proton $\left({ }^{1} \mathrm{H}\right)$ and carbon- $13\left({ }^{13} \mathrm{C}\right)$ nuclear magnetic resonance (NMR) spectroscopy and electrospray ionization mass spectrometry (ESI-MS).
\end{abstract}

Keywords: oxazin, pyrane, quinolinone, heterocyclization, heteroannulation

\section{Introduction}

The synthesis of many new different polycyclic heterocycles has received a large interest in research on organic, combinatorial, and medicinal chemistry. ${ }^{1-5}$ Oxazines heterocycles are special arresting group of compounds known to show effective biological and medicinal properties. ${ }^{6}$ The 1,4 -oxazine scaffold is a structural subunit of many naturally occurring and synthetic bioactive compounds and have many biological activities such as anti-inflammatory, ${ }^{7}$ antiulcer, ${ }^{8}$ antipyretic, ${ }^{9}$ antihypertensive, ${ }^{10}$ antifungal, ${ }^{11}$ antirheumatic agents. ${ }^{12}$ Pyrano[3,2-c]quinolinones skeletons, including both quinolone ring and pyran moiety, have received much attention due to their various biological properties. ${ }^{13-16}$

Despite this extensive body of published work on the biological activities of these two types of heterocycles, we did not find any literature reports pointing to the heteroannulation of an oxazine nucleus with the pyranoquinolone moiety in one fused molecular frame. Rings annulated to the pyranoquinolinone units showed potential medicinal properties such as antibacterial, ${ }^{17}$ anticoagulant, ${ }^{18}$ antitumor, ${ }^{19}$ and microtubule-targeting agents. ${ }^{14}$ Prompted by the encouraging literature precedent we embarked on the synthesis of this fused heteroannulated system. We recently reported the preparation of synthetically valuable 3 -amino4-hydroxypyranoquinolinone (1) as a versatile precursor for hetrocyclization reaction of pyranoquinolinone. ${ }^{20}$ Herein we utilize 3-amino-4-hydroxypyrano[3,2-c] quinoline-

*e-mail: mmhassan121@yahoo.com
2,5(6H)-dione (1) and 3-nitro-4-hydroxypyrano[3,2-c] quinoline-2,5(6H)-dione (10) to get a new skeleton of pyranoquinolinones incorporating 1,4-oxazine ring at face $c$, with the hope that these compounds turn out to be biologically active.

\section{Experimental}

Melting points (mp) were recorded on Sanyo Gallenkamp MPD 350-BM 3.5 melting point apparatus. Thermo Nicolet Nexus 470 FTIR spectrometer was used for infrared (IR) analyses. Proton $\left({ }^{1} \mathrm{H}\right)$ and carbon- $13\left({ }^{13} \mathrm{C}\right)$ nuclear magnetic resonance (NMR) spectra were recorded at room temperature in base-filtered DMSO- $d_{6}$ or $\mathrm{CDCl}_{3}$ as a solvent and tetramethylsilane (TMS) as an internal standard on a Bruker Avance III- $400 \mathrm{MHz}$ or Varian-400 MHz instrument operating at $400 \mathrm{MHz}$ for protons and $101 \mathrm{MHz}$ for carbon. Elemental microanalyses were performed on PerkinElmer CHN 2400II at the Chemical War department, Ministry of Defense, Cairo, Egypt. Electrospray ionization mass spectrometry (ESI-MS) was recorded on a Micromass LC-ZMD single quadrupole liquid chromatography mass spectrometer. Analytical thin layer chromatography (TLC) was performed on aluminum-backed $0.2 \mathrm{~mm}$ thick silica gel 60 F254 plates. Eluted plates were visualized using a $254 \mathrm{~nm} \mathrm{UV}$ lamp. Column chromatographic separations were carried out with silica gel 60 (40-63 microns) as the stationary phase and using the analytical reagent (AR) or high performance liquid chromatography (HPLC) grade solvents as indicated. Starting materials, reagents, drying agents and 
other inorganic salts were generally commercially available and were used as supplied.

\section{6-Butyl-2-hydroxy-5H,6H-[1,4]oxazino[2',3':4,5]pyrano} [3,2-c]quinoline-3,5,12-trione (2)

A mixture of compound $\mathbf{1}(3 \mathrm{~g}, 10 \mathrm{mmol})$ and oxalyl chloride $(0.9 \mathrm{~mL}, 2 \mathrm{mmol})$, in dichloromethane $(50 \mathrm{~mL})$, was heated under reflux for $2 \mathrm{~h}$. The progress of the reaction was monitored by TLC. After completion of the reaction, the reaction mixture was cooled to room temperature. The precipitated solid was filtered off, air dried, and recrystallized from ethanol $(\mathrm{EtOH})$ to give compound $\mathbf{2}$ as yellow crystals in $65 \%$ yield; $\mathrm{mp}>300{ }^{\circ} \mathrm{C}$; IR $(\mathrm{KBr}) \mathrm{v} / \mathrm{cm}^{-1}$ $3414(\mathrm{OH}), 3094\left(\mathrm{CH}_{\text {aromatic }}\right), 2955,2915,2846\left(\mathrm{CH}_{\text {aliphatic }}\right)$, $1748\left(\mathrm{C}=\mathrm{O}_{\alpha \text {-pyrone }}\right), 1702\left(\mathrm{O}-\mathrm{C}=\mathrm{O}_{\text {oxazine }}\right), 1650\left(\mathrm{~N}-\mathrm{C}=\mathrm{O}_{\text {oxazine }}\right)$, $1629\left(\mathrm{C}=\mathrm{O}_{\text {quinolone }}\right), 1610(\mathrm{C}=\mathrm{N}), 1570\left(\mathrm{C}=\mathrm{C}_{\text {aromatic }}\right) ;{ }^{1} \mathrm{H} \mathrm{NMR}$ (400 MHz, DMSO- $d_{6}$ ) $\delta 0.94\left(\mathrm{t}, J 7.7 \mathrm{~Hz}, 3 \mathrm{H}, \mathrm{C} 4{ }^{\prime}-\mathrm{Hs}\right)$, 1.42-1.46 (m, 2H, C3'-Hs), 1.54-1.65 (m, 2H, C2'-Hs), 4.33 (t, 2H, J $7.6 \mathrm{~Hz}, \mathrm{C} 1$ '- $-\mathrm{Hs}$ ), 5.45 (s, 0.5H, NH), 7.49 (dd, J 8.1, 1.4 Hz, 1H, C9-H), 7.79-7.84 (m, 2H, C7-H and C8-H), 8.06 (d, 1H, J 8.5 Hz, C10-H), 13.2 (s, 0.5H, OH); ${ }^{13} \mathrm{C}$ NMR (101 MHz, DMSO- $d_{6}$ ) $\delta 14.1$ (C4'), 19.9 (C3'), 29.6 (C2'), 41.0 (C1'), 101.7, 114.0, 114.9, 116.4, 124.4, $124.8,132.2,134.9,136.1,144.5,151.5,159.0,162.8$, 163.5; ESI-MS $m / z, 355.1[\mathrm{M}+\mathrm{H}]^{+}$and $377.1[\mathrm{M}+\mathrm{Na}]^{+}$; anal. calcd. for $\mathrm{C}_{18} \mathrm{H}_{14} \mathrm{~N}_{2} \mathrm{O}_{6}$ (354.32): $\mathrm{C} \mathrm{61.02,} \mathrm{H} 3.98$, N 7.91, found: C 61.01, H 3.90, N 7.89\%.

6-Butyl-3-(3-chloro-pyridin-2-ylamino)-4-hydroxypyrano [3,2-c]quinolone-2,5(6H)dione (3)

To a solution of compound $\mathbf{1}(3 \mathrm{~g}, 10 \mathrm{mmol})$ in tetrahydrofuran (THF) $(25 \mathrm{~mL})$, an equivalent amount of 2,3-dichloropyridine (1.48 g, $10 \mathrm{~mL})$, was added and the reaction mixture was stirred at room temperature until the reaction was completely judged by TLC $(6 \mathrm{~h})$. The reaction mixture was poured on ice $(200 \mathrm{~g})$. The obtained precipitate was filtered, washed with water $(3 \times 10 \mathrm{~mL})$, dried and crystallized from ethanol to furnish $\mathbf{3}$ as yellow crystals in $50 \%$ yield; mp $178-179{ }^{\circ} \mathrm{C}$; IR $(\mathrm{KBr}) \mathrm{v} / \mathrm{cm}^{-1}$ $3458(\mathrm{OH}), 3342,3220(\mathrm{NH}), 3086\left(\mathrm{CH}_{\text {aromatic }}\right), 2930,2918$, $2849\left(\mathrm{CH}_{\text {aliphatic }}\right), 1745\left(\mathrm{C}=\mathrm{O}_{\alpha \text {-pyrone }}\right), 1676\left(\mathrm{C}=\mathrm{O}_{\text {quinolone }}\right), 1614$ $(\mathrm{C}=\mathrm{N}), 1577\left(\mathrm{C}=\mathrm{C}_{\text {aromatic }}\right) ;{ }^{1} \mathrm{H}$ NMR $\left(400 \mathrm{MHz}, \mathrm{DMSO}-d_{6}\right)$ $\delta 0.95\left(\mathrm{t}, 3 \mathrm{H}, J 8.00 \mathrm{~Hz}, \mathrm{C} 4{ }^{\prime}-\mathrm{Hs}\right), 1.42-1.47(\mathrm{~m}, 2 \mathrm{H}$, C3'-Hs), 1.64-1.69 (m, 2H, C2'-Hs), 4.34 (t, 2H, J 8.00 Hz, C1'-Hs), 7.31 (t, 1H, J $\left.8.00 \mathrm{~Hz}, \mathrm{CH}_{\text {aromatic }}\right), 7.52(\mathrm{t}, 1 \mathrm{H}$, $\left.J 8.00 \mathrm{~Hz}, \mathrm{CH}_{\text {aromatic }}\right), 7.74$ (d, $1 \mathrm{H}, J 8.00 \mathrm{~Hz}, \mathrm{CH}_{\text {aromatic }}$ ), 7.79 (t, 1H, J $\left.8.00 \mathrm{~Hz}, \mathrm{CH}_{\text {aromatic }}\right), 7.88$ (d, 1H, J $8.00 \mathrm{~Hz}$, $\mathrm{CH}_{\text {aromatic }}$ ), 8.00 (t, $\left.1 \mathrm{H}, J 8.00 \mathrm{~Hz}, \mathrm{CH}_{\text {aromatic }}\right), 8.17$ (d, $1 \mathrm{H}$, $J$ 8.00, 1.2 Hz, C10-H), 9.27 (s, 1H, NH), 13.98 (s, 1H, $\mathrm{OH}) ;{ }^{13} \mathrm{C} \mathrm{NMR}\left(101 \mathrm{MHz}, \mathrm{DMSO}-d_{6}\right) \delta 13.9$ (s, C4'), 19.9 (s, C3'), 29.3 (s, C2'), 42.3 (s, C1'), 99.8, 102.6,
$113.6,115.5,115.9,116.5,123.0,124.2,134.3,134.6$, $137.9,157.5,158.9,159.5,160.0,163.2$, 163.8; ESI-MS $\mathrm{m} / z, 412.9[\mathrm{M}+\mathrm{H}]^{+}$and $434.7[\mathrm{M}+\mathrm{Na}]^{+}$; anal. calcd. for $\mathrm{C}_{21} \mathrm{H}_{18} \mathrm{ClN}_{3} \mathrm{O}_{4}$ (411.85): C 61.24, $\mathrm{H} 4.41, \mathrm{~N} 10.20$, found: C 61.11, H 4.39, N 10.18\%.

14-Butyl-7,14-dihydro-pyrido[3",2":5',6']1,4-oxazino [2',3':4,5]pyrano[3,2-c]quinoline-6,13-dione (5)

A mixture of compound $4(4.11 \mathrm{~g}, 10 \mathrm{mmol})$ and dimethylformamide (DMF) $(25 \mathrm{~mL})$ containing sodium hydride $(\mathrm{NaH})(0.24 \mathrm{~g})$ was heated under reflux for $6 \mathrm{~h}$. Progress of the reaction was tested by TLC. The reaction mixture was filtered and the filtrate poured on ice (200 g). The obtained solid was filtered, washed with water $(3 \times 20 \mathrm{~mL})$, then diethyl ether $(3 \times 20 \mathrm{~mL})$ and crystallized from acetic acid to give compound $\mathbf{5}$ as pale yellow crystals in $72 \%$ yield; $\mathrm{mp}>300{ }^{\circ} \mathrm{C}$; IR $(\mathrm{KBr}) \mathrm{v} / \mathrm{cm}^{-1} 3266(\mathrm{NH})$, 2959, 2930, 2859 ( $\left.\mathrm{CH}_{\text {aliphatic }}\right), 1748\left(\mathrm{C}=\mathrm{O}_{\alpha \text {-pyrone }}\right), 1677$ $\left(\mathrm{C}=\mathrm{O}_{\text {quinolone }}\right), 1639(\mathrm{C}=\mathrm{N}), 1606\left(\mathrm{C}=\mathrm{C}_{\text {aromatic }}\right) ;{ }^{1} \mathrm{H}$ NMR (400 MHz, DMSO- $d_{6}$ ) $\delta 0.94$ (t, 3H, J 7.3 Hz, C4'-Hs), 1.40-1.50 (m, 2H, C3'-Hs), 1.64-1.72 (m, 2H, C2'-Hs), 4.35 (t, 2H, J 7.8 Hz, C1'-Hs), 7.32-7.47 (m, 2H, C3-H and $\left.\mathrm{H}_{\text {pyrido }}\right), 7.53$ (d, $\left.1 \mathrm{H}, J 7.6 \mathrm{~Hz}, \mathrm{C} 1-\mathrm{H}\right), 7.74$ (dd, 1H, J 8.1, $1.1 \mathrm{~Hz}, \mathrm{C} 2-\mathrm{H}), 7.87$ (t, $1 \mathrm{H}, J 8.1 \mathrm{~Hz}, \mathrm{H}_{\text {pyrido }}$ ), 7.97-8.19 (m, 2H, C4-H and $\mathrm{H}_{\text {pyrido }}$ ), 9.59 (s, $\left.1 \mathrm{H}, \mathrm{NH}\right) ;{ }^{13} \mathrm{C} \mathrm{NMR}$ (101 MHz, DMSO- $d_{6}$ ) $\delta 14.1$ (C4'), 19.9 (C3'), 29.1 (C2'), 42.1 (C1'), 100.9, 112.4, 113.7, 114.1, 114.4, 116.6, 119.4, $122.7,124.2,124.6,132.1,134.1,134.9,151.2,152.1$, 159.3, 163.9; ESI-MS $m / z, 376.4[\mathrm{M}+\mathrm{H}]^{+}$and 398.1 $[\mathrm{M}+\mathrm{Na}]^{+}$; anal. calcd. for $\mathrm{C}_{21} \mathrm{H}_{17} \mathrm{~N}_{3} \mathrm{O}_{4}$ (375.39): $\mathrm{C}$ 67.19, H 4.56, N 11.19, found: C 67.01, H 4.70, N 11.08\%.

16-Butyl-7,16-dihydro-quinoxalino[2",3":5',6']-1,4-oxazino [2',3':4,5]pyrano[3,2-c]quinoline-6,15-dione (6)

A mixture of compounds $1(3 \mathrm{~g}, 10 \mathrm{mmol})$ and 2,3-dichloroquinoxaline (1.98 g, $10 \mathrm{~mL})$ with DMF $(50 \mathrm{~mL})$ containing few drops of triethanolamine (TEA) was heated under reflux for $4 \mathrm{~h}$. During this time the targeted compound $\mathbf{6}$ was precipitated. The reaction mixture was cooled to room temperature. The obtained solid was filtered, washed with ethanol $(3 \times 10 \mathrm{~mL})$ and crystallized from acetic acid to give the corresponding compound 6 as pale yellow crystals in $69 \%$ yield; $\mathrm{mp}>300{ }^{\circ} \mathrm{C}$; IR $(\mathrm{KBr}) v / \mathrm{cm}^{-1} 3292(\mathrm{NH}), 2956,2932,2876\left(\mathrm{CH}_{\text {aliphatic }}\right)$, $1728\left(\mathrm{C}=\mathrm{O}_{\alpha \text {-pyrone }}\right), 1679\left(\mathrm{C}=\mathrm{O}_{\text {quinolone }}\right), 1613(\mathrm{C}=\mathrm{N}), 1576$ $\left(\mathrm{C}=\mathrm{C}_{\text {aromatic }}\right) ;{ }^{1} \mathrm{H}$ NMR $\left(400 \mathrm{MHz}, \mathrm{DMSO}-d_{6}\right) \delta 0.92(\mathrm{t}$, 3H, J 7.3 Hz, C4'-Hs), 1.41-1.49 (m, 2H, C3'-Hs), 1.611.70 (m, 2H, C2'-Hs), 4.41 (t, 2H, J 7.8 Hz, C1'-Hs), $7.01\left(\mathrm{~d}, 1 \mathrm{H}, J 8.1 \mathrm{~Hz}, \mathrm{H}_{\text {benzo }}\right), 7.22-7.34(\mathrm{~m}, 2 \mathrm{H}, \mathrm{C} 3-\mathrm{H}$ and $\left.\mathrm{H}_{\text {benzo }}\right), 7.50-7.59\left(\mathrm{~m}, 2 \mathrm{H}, \mathrm{C} 1-\mathrm{H}\right.$ and $\left.\mathrm{H}_{\text {benzo }}\right), 7.70-7.82$ (m, 2H, C2-H and $\mathrm{H}_{\text {benzo }}$ ), 8.20 (d, $\left.1 \mathrm{H}, J 8.0 \mathrm{~Hz}, \mathrm{C} 4-\mathrm{H}\right)$, 
$9.01(\mathrm{~s}, 1 \mathrm{H}, \mathrm{NH}) ;{ }^{13} \mathrm{C}$ NMR $\left(101 \mathrm{MHz}, \mathrm{DMSO}-d_{6}\right) \delta 13.4$ (C4'), 21.0 (C3'), 31.1 (C2'), 38.0 (C1'), 98.3, 104.9, 114.6, $114.9,115.6,115.9,116.5,121.4,122.6,123.5,125.6$, $131.7,135.8,140.3,141.8,159.2,160.7,161.4,162.8$, 173.4; ESI-MS $m / z, 427.5[\mathrm{M}+\mathrm{H}]^{+}$and $449.4[\mathrm{M}+\mathrm{Na}]^{+}$; anal. calcd. for $\mathrm{C}_{24} \mathrm{H}_{18} \mathrm{~N}_{4} \mathrm{O}_{4}$ (426.44): C 67.60, H 4.25, $\mathrm{N}$ 13.14, found: C 67.52, H 4.29, N 13.10\%.

\section{6-Butyl-3-phenyl[1,4]oxazino[2', 3':4,5]pyrano[3,2-c]} quinoline-5,12(6H)-dione (7)

A mixture of compound $1(3 \mathrm{~g}, 10 \mathrm{mmol})$ and phenacyl bromide $(2 \mathrm{~g}, 10 \mathrm{mmol})$, in DMF $(50 \mathrm{~mL})$ containing few drops of TEA, was heated under reflux for $3 \mathrm{~h}$. After cooling, the reaction mixture was poured onto ice water. The precipitated solid was filtered off, washed with water, air dried, and crystallized from DMF to give compound 7 as pale brown crystals in $63 \%$ yield; $\mathrm{mp}>300^{\circ} \mathrm{C}$; IR $(\mathrm{KBr})$ $v / \mathrm{cm}^{-1} 3203(\mathrm{NH}), 2958,2928,2876\left(\mathrm{CH}_{\text {aliphatic }}\right), 1738$ $\left(\mathrm{C}=\mathrm{O}_{\alpha \text {-pyrone }}\right), 1676\left(\mathrm{C}=\mathrm{O}_{\text {quinolone }}\right), 1613\left(\mathrm{C}=\mathrm{C}_{\text {aromatic }}\right) ;{ }^{1} \mathrm{H}$ NMR $\left(400 \mathrm{MHz}, \mathrm{DMSO}-d_{6}\right) \delta 0.90\left(\mathrm{t}, J 7.3 \mathrm{~Hz}, 3 \mathrm{H}, \mathrm{C} 4^{\prime}-\mathrm{Hs}\right)$, 1.43-1.40 (m, 2H, C3'-Hs), 1.59-1.63 (m, 2H, C2'-Hs), 4.33 (t, $2 \mathrm{H}, J 7.8 \mathrm{~Hz}, \mathrm{C} 1$ '-Hs), 7.11 (t, $\left.1 \mathrm{H}, J 7.7 \mathrm{~Hz}, \mathrm{H}_{\text {phenyl }}\right)$, $7.29\left(\mathrm{~s}, 1 \mathrm{H}, \mathrm{CH}_{\text {oxazine }}\right), 7.35-7.42\left(\mathrm{~m}, 2 \mathrm{H}, \mathrm{C} 9-\mathrm{H}\right.$ and $\left.\mathrm{H}_{\text {pheny }}\right)$, 7.50-7.59 (m, $3 \mathrm{H}, 7-\mathrm{H}$ and $\left.2 \mathrm{H}_{\text {pheny }}\right), 7.80-7.89(\mathrm{~m}, 2 \mathrm{H}, \mathrm{C} 8-\mathrm{H}$ and $\left.\mathrm{H}_{\text {phenyl }}\right), 8.16(\mathrm{~d}, 1 \mathrm{H}, J 8.1 \mathrm{~Hz}, \mathrm{C} 10-\mathrm{H}), 8.94(\mathrm{~s}, 1 \mathrm{H}, \mathrm{NH})$; ${ }^{13} \mathrm{C}$ NMR (101 MHz, DMSO- $d_{6}$ ) $\delta 13.1$ (C4'), 19.9 (C3'), 31.1 (C2'), 42.3 (C1'), 97.2, 104.1, 108.9, 109.9, 115.2, $115.5,116.4,121.2,122.6,123.4,123.9,124.6,126.2$, 132.9, 133.8, 139.1, 141.6, 158.4, 161.5, 162.7; ESI-MS $\mathrm{m} / z, 401.5[\mathrm{M}+\mathrm{H}]^{+}$and $423.3\left[\mathrm{M}+\mathrm{Na}^{+}\right.$; anal. calcd for $\mathrm{C}_{24} \mathrm{H}_{20} \mathrm{~N}_{2} \mathrm{O}_{4}$ (400.44): C 71.99, H 5.03, N 7.00, found: C 71.71, H 5.00, N 6.98\%.

Ethyl[(6-butyl-4-hydroxy-2,5-dioxo-5,6-dihydro-2 $\mathrm{H}$-pyrano [3,2-c]quinolin-3-yl)amino]acetate (8)

A mixture of compound 1 ( $3 \mathrm{~g}, 10 \mathrm{mmol})$ and dry acetonitrile $(50 \mathrm{~mL})$ containing $\mathrm{NaH}(0.24 \mathrm{~g}, 10 \mathrm{mmol})$ was stirred at room temperature for $30 \mathrm{~min}$. During this time, ethyl bromoacetate $(1.11 \mathrm{~mL}, 10 \mathrm{mmol})$ was added dropwise to the previous mixture. The reaction mixture was heated under reflux for $6 \mathrm{~h}$ and it was monitored by TLC. At the end of the reaction, the reaction mixture was filtered on hot and the filtrate was poured on ice $(200 \mathrm{~g})$. The resulting solid was chromatographed on a silica gel column eluted with ethyl acetate/hexane (6:4, v:v) to give compound $\mathbf{8}$ as brown crystals in $55 \%$ yield; mp $164-166^{\circ} \mathrm{C}$; IR $(\mathrm{KBr})$ $v / \mathrm{cm}^{-1} 3427(\mathrm{OH}), 3094(\mathrm{NH}), 2959,2912,2848\left(\mathrm{CH}_{\text {aliphatic }}\right)$, $1744\left(\mathrm{C}=\mathrm{O}_{\text {ester }}\right), 1703\left(\mathrm{C}=\mathrm{O}_{\alpha \text {-pyrone }}\right), 1643\left(\mathrm{C}=\mathrm{O}_{\text {quinolone }}\right), 1600$ $\left(\mathrm{C}=\mathrm{C}_{\text {aromatic }}\right) ;{ }^{1} \mathrm{H}$ NMR $\left(400 \mathrm{MHz}, \mathrm{DMSO}-d_{6}\right) \delta 0.90$ (t, $\left.J 7.5 \mathrm{~Hz}, 3 \mathrm{H}, \mathrm{C} 4{ }^{\prime}-\mathrm{Hs}\right), 1.13$ (t, $J 6.4 \mathrm{~Hz}, 3 \mathrm{H}, \mathrm{CH}_{3 \text { ester }}$ ), 1.41-1.43 (m, 2H, C3'-Hs), 1.60-1.65 (m, 2H, C2'-Hs), 4.31 (t, 2H, J $8 \mathrm{~Hz}, \mathrm{C1}$ '-Hs), 4.42 (q, 2H, J $6.4 \mathrm{~Hz}, \mathrm{CH}_{2 \text { ester }}$ ), 5.23 (s, $2 \mathrm{H}, \mathrm{NCH}_{2} \mathrm{CO}$ ), 6.16 (s, 1H, NH), 7.35 (dd, $1 \mathrm{H}, J$ 8.1, $1.5 \mathrm{~Hz}, \mathrm{C} 9-\mathrm{H}), 7.50$ (d, 1H, J 8.1 Hz, C7-H), 7.80 (dd, $1 \mathrm{H}, J$ 8.1, $1.5 \mathrm{~Hz}, \mathrm{C} 8-\mathrm{H}), 8.16$ (d, 1H, J $8.0 \mathrm{~Hz}, \mathrm{C} 10-\mathrm{H})$, $13.24(\mathrm{~s}, 1 \mathrm{H}, \mathrm{OH}) ;{ }^{13} \mathrm{C}$ NMR (101 MHz, DMSO- $\left.d_{6}\right) \delta 14.1$

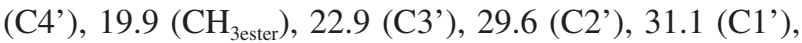
$40.4\left(\mathrm{NCH}_{2} \mathrm{CO}\right), 42.3\left(\mathrm{CH}_{2 \text { ester }}\right), 99.9,102.2,113.5,116.7$, 124.1, 124.7, 134.4, 136.0, 156.9, 159.3, 162.9, 169.0, $206.9\left(\mathrm{C}=\mathrm{O}_{\text {ester }}\right)$; ESI-MS $\mathrm{m} / z, 387.5[\mathrm{M}+\mathrm{H}]^{+}$and 409.3 $[\mathrm{M}+\mathrm{Na}]^{+}$; anal. calcd. for $\mathrm{C}_{20} \mathrm{H}_{22} \mathrm{~N}_{2} \mathrm{O}_{6}(386.41)$ : C 62.17, H 5.74, N 7.25, found: C 62.11, H 5.70, N 7.08\%.

26-Butyl-3-hydroxy[1,4]oxazino[2',3':4,5]pyrano[3,2-c] quinoline-5,12(6H)-dione (9)

Method (i): a mixture of compound 1 ( $3 \mathrm{~g}, 10 \mathrm{mmol})$ and chloroacetic acid $(0.85 \mathrm{~g}, 10 \mathrm{mmol})$, in DMF $(50 \mathrm{~mL})$ containing few drops of TEA, was heated under reflux for $2 \mathrm{~h}$. The progress of the reaction was monitored by TLC. After completion of reaction, the reaction mixture was poured onto ice $(200 \mathrm{~g})$. The precipitated solid was filtered off, washed with water $(3 \times 20 \mathrm{~mL})$, air dried, and crystallized from DMF to give compound $\mathbf{9}$ as pale yellow crystals in yield $48 \%$; $\mathrm{mp}>300{ }^{\circ} \mathrm{C}$.

Method (ii): to a mixture of $8(3.86 \mathrm{~g}, 10 \mathrm{mmol})$ in glacial acetic acid $(80 \%)(40 \mathrm{~mL})$, acetic anhydride $(20 \%)(10 \mathrm{~mL})$ was added then heated under reflux until the reaction was completely analysed by TLC $(8 \mathrm{~h})$. The reaction mixture cooled to room temperature and poured on ice $(200 \mathrm{~g})$. The formed precipitate was filtered, washed with water $(3 \times 20 \mathrm{~mL})$, dried and crystallized from DMF to give compound 9 as pale yellow crystals in $80 \%$ yield; $\mathrm{mp}>300^{\circ} \mathrm{C}$; IR $(\mathrm{KBr}) \mathrm{v} / \mathrm{cm}^{-1} 3342(\mathrm{OH}), 3156$ $\left(\mathrm{CH}_{\text {aromatic }}\right), 2967,2928,2850\left(\mathrm{CH}_{\text {aliphatic }}\right), 1748\left(\mathrm{C}=\mathrm{O}_{\alpha \text {-pyrone }}\right)$, $1679\left(\mathrm{C}=\mathrm{O}_{\text {quinolone }}\right), 1617\left(\mathrm{C}=\mathrm{C}_{\text {aromatic }}\right) ;{ }^{1} \mathrm{H} \mathrm{NMR}(400 \mathrm{MHz}$, DMSO- $\left.d_{6}\right) \delta 0.94$ (t, 3H, $J 7.5 \mathrm{~Hz}, \mathrm{C} 4$ '-Hs), 1.42-1.46 (m, 2H, C3'-Hs), 1.67 (m, 2H, C2'-Hs), 4.33 (t, 2H, J8.0 Hz, C1'-Hs), 5.79 (s, 1H, $\left.\mathrm{CH}_{\text {2oxazine }}\right), 6.48(\mathrm{~s}, 1 \mathrm{H}, \mathrm{NH}), 7.33$ (s, $0.5 \mathrm{H}, \mathrm{H}-2_{\text {oxazine }}$ ), 7.53 (dd, $\left.1 \mathrm{H}, J 8.1,1.2 \mathrm{~Hz}, \mathrm{C} 9-\mathrm{H}\right)$, 7.75-7.86 (m, 2H, C7-H and C8-H), $8.14(\mathrm{~d}, 1 \mathrm{H}, J 8.0 \mathrm{~Hz}$, C10-H), $13.65(\mathrm{~s}, 1 \mathrm{H}, \mathrm{OH}) ;{ }^{13} \mathrm{C}$ NMR (101 MHz, DMSO- $d_{6}$ ) d 13.4 (C4'), 19.9 (C3'), 31.1 (C2'), 36.0 (C1'), 113.4, $114.7,121.4,122.8,123.9,126.3,130.1,132.8,139.6$, 150.7, 162.7, 175.5; ESI-MS $m / z, 341.3[\mathrm{M}+\mathrm{H}]^{+}$and $363.2[\mathrm{M}+\mathrm{Na}]^{+}$; anal. calcd. for $\mathrm{C}_{18} \mathrm{H}_{16} \mathrm{~N}_{2} \mathrm{O}_{5}$ (340.34): $\mathrm{C}$ 63.53, H 4.74, N 8.23, found: C 63.51, H 4.70, N 8.12\%.

(6-Butyl-3-nitro-2,5-dioxo-5,6-dihydro-2H-pyrano[3,2-c] quinolin-4-yloxyl)acetonitrile (11)

A mixture of compound $\mathbf{1 0}(3.3 \mathrm{~g}, 10 \mathrm{mmol})$ and dry acetonitrile $(50 \mathrm{~mL})$ containing $\mathrm{NaH}(0.24 \mathrm{~g}, 10 \mathrm{mmol})$ was stirred at room temperature for $30 \mathrm{~min}$. During this 
time, bromoacetonitrile $(1.2 \mathrm{~mL}, 10 \mathrm{mmol})$ was added dropwise and the reaction mixture was heated under reflux for $12 \mathrm{~h}$ and it was monitored by TLC. At the end of the reaction, the reaction mixture was poured on ice (200 g). The obtained dark brown solid was filtered, washed with water $(3 \times 20 \mathrm{~mL})$, dried and rechromatographed on silica gel column eluting with a mixture of ethyl acetate/hexane in the ratio of 9:1 (v:v) to give compound $\mathbf{1 1}$ as brown crystals in 47\% yield; $\mathrm{mp} 180-182^{\circ} \mathrm{C}$; IR $(\mathrm{KBr}) \mathrm{v} / \mathrm{cm}^{-1} 2948,2923$, $2850\left(\mathrm{CH}_{\text {aliphatic }}\right), 2201(\mathrm{C} \equiv \mathrm{N}), 1719\left(\mathrm{C}=\mathrm{O}_{\alpha \text {-pyrone }}\right), 1645$ $\left(\mathrm{C}=\mathrm{O}_{\text {quinolone }}\right) ;{ }^{1} \mathrm{H} \mathrm{NMR}\left(400 \mathrm{MHz}, \mathrm{CDCl}_{3}\right) \delta 1.2(\mathrm{t}, J 7.7 \mathrm{~Hz}$, 3H, C4'-Hs), 1.50-1.54 (m, 2H, C3'-Hs), 1.75-1.78 (m, 2H, C2'-Hs), 4.21 (s, 2H, CH 2acetonitrile ), 4.36 (t, 2H, J $7.8 \mathrm{~Hz}$, C1'-Hs), 7.51 (dd, 1H, J 8.0, 1.1 Hz, C9-H), 7.79-7.86 (m, 2H, C7-H and C8-H), 8.30 (d, 1H, J 8.0 Hz, C10-H); ${ }^{13} \mathrm{C}$ NMR (101 MHz, DMSO- $d_{6}$ ) $\delta 13.3$ (C4'), 18.9 (C3'), 37.1 (C2'), 40.5 (C1'), $56.4\left(\mathrm{CH}_{2 \text { acetonitrile }}\right), 112.2,112.6$, $115.5,119.6,123.0,124.2,135.2,139.9,150.3,157.0$, 159.8, 161.9, 169.2; ESI-MS $m / z, 370.4[\mathrm{M}+\mathrm{H}]^{+}$and 392.4 $[\mathrm{M}+\mathrm{Na}]^{+}$; anal. calcd. for $\mathrm{C}_{18} \mathrm{H}_{15} \mathrm{~N}_{3} \mathrm{O}_{6}$ (369.34): C 58.54, H 4.09, N 11.38, found: C 58.50, H 4.07, N 11.28\%.

6-Butyl-2-hydroxy-[1,4]oxazino[2',3':4,5]pyrano[3,2-c] quinolone-5,12(6H)-dione (12)

Method (i): to a stirred solution of $\mathbf{1 1}(3.69 \mathrm{~g}, 10 \mathrm{mmol})$ in acetic acid $(50 \mathrm{~mL})$, powdered iron $(\mathrm{Fe})(3.35 \mathrm{~g}, 60 \mathrm{mmol})$ was added and the reaction mixture was stirred for $5 \mathrm{~min}$ at room temperature and immediately it was cooled to $0{ }^{\circ} \mathrm{C}$. The reaction mixture was stirred for $2 \mathrm{~min}$ in ice cold water bath (slightly exothermic), then it was refluxed for $2.5 \mathrm{~h}$. The progress of the reaction was monitored by TLC. After completion of the reaction, the reaction mixture was cooled to room temperature and the acetic acid was removed under reduced pressure, ethyl acetate (EtOAc) $(150 \mathrm{~mL})$ was added and was stirred for $2 \mathrm{~min}$, and then filtered to remove any iron impurities. The insoluble iron residue was washed with EtOAc $(100 \mathrm{~mL})$. The filtrate and washings were combined and dried over anhydrous magnesium sulfate $\left(\mathrm{MgSO}_{4}\right)$. The solvent was removed under reduced pressure and the crude solid obtained was then chromatographed on silica gel column eluting with a mixture of ethyl acetate/hexane $(9: 1, \mathrm{v}: \mathrm{v}$, ratio) to give after evaporation of the identical fractions monitored by TLC the pure product $\mathbf{1 2}$ as yellow crystals in $60 \%$ yield; mp 210-212 ${ }^{\circ} \mathrm{C}$.

Method (ii): a mixture of compound 1 (3 g, $10 \mathrm{mmol})$ and DMF (50 mL) containing $\mathrm{NaH}(0.24 \mathrm{~g}, 10 \mathrm{mmol})$ was stirred at room temperature for $30 \mathrm{~min}$. During this time, one molar ratio $(0.8 \mathrm{~mL}, 10 \mathrm{mmol})$ of chloroacetylchloride, was added dropwise and the reaction mixture was heated under reflux for $12 \mathrm{~h}$ and it was monitored by TLC. At the end of the reaction, the reaction mixture was poured on ice (200 g). The obtained solid was filtered, washed by water $(3 \times 20 \mathrm{~mL})$, dried and crystallized from acetic acid $(\mathrm{AcOH})$ to give compound $\mathbf{1 2}$ in $61 \%$ yield; $\mathrm{mp} 210-211^{\circ} \mathrm{C}$; IR (KBr) v / cm $13413(\mathrm{OH}), 3280,3105(\mathrm{NH}), 3049$ $\left(\mathrm{CH}_{\text {aromatic }}\right), 2954,2848\left(\mathrm{CH}_{\text {aliphatic }}\right), 1715\left(\mathrm{C}=\mathrm{O}_{\alpha \text {-pyrone }}\right), 1639$ $\left(\mathrm{C}=\mathrm{O}_{\text {quinolone }}\right), 1610(\mathrm{C}=\mathrm{N}), 1582\left(\mathrm{C}=\mathrm{C}_{\text {aromatic }}\right) ;{ }^{1} \mathrm{H}$ NMR (400 $\left.\mathrm{MHz}, \mathrm{CDCl}_{3}\right) \delta 0.99(\mathrm{t}, 3 \mathrm{H}, J 7.3 \mathrm{~Hz}, \mathrm{C} 4$ '-Hs), 1.45-1.49 (m, 2H, C3'-Hs), 1.73-1.76 (m, 2H, C2'-Hs), 4.30 (t, 2H, J $8 \mathrm{~Hz}, \mathrm{C} 1$ '-Hs), 5.58 (s, 1H, NH), 7.38-7.48 (m, 2H, C9-H and C7-H), $7.75(\mathrm{dd}, 1 \mathrm{H}, J$ 8.0, $1.2 \mathrm{~Hz}$, C8-H), 8.23 (d, 1H, J 8.0 Hz, C10-H), 8.98 (s, 1H, $\mathrm{CH}_{\text {oxazine }}$ ), 13.22 (s, 1H, OH); $\left.{ }^{13} \mathrm{C} \mathrm{NMR} \mathrm{(101} \mathrm{MHz,} \mathrm{CDCl}_{3}\right) \delta 13.7$ (C4'), 20.1 (C3'), 29.5 (C2'), 42.5 (C1'), 113.3, 113.7, $114.9,115.1,123.5,124.9,129.6,133.3,138.0,146.2$, 149.2, 157.4, 162.5, 163.1; ESI-MS $m / z, 341.4[\mathrm{M}+\mathrm{H}]^{+}$ and $363.3[\mathrm{M}+\mathrm{Na}]^{+}$; anal. calcd. for $\mathrm{C}_{18} \mathrm{H}_{16} \mathrm{~N}_{2} \mathrm{O}_{5}(340.34)$ : C 63.53, H 4.74, N 8.23, found: C 63.50, H 4.68, N 8.09\%.

\section{6-Butyl-4-chloro-3-nitropyrano[3,2-c]quinoline-} 2,5(6H)-dione (13)

A solution of compound $10(3.3 \mathrm{~g}, 10 \mathrm{mmol})$ in phosphoryl chloride $(15.2 \mathrm{~mL}, 100 \mathrm{mmol})$ was refluxed for $2 \mathrm{~h}$. The excess solvent was removed by distillation and the residue poured on ice water $(100 \mathrm{~mL})$. The precipitate so formed was filtered, washed with water, dried and crystallized from EtOH to give compound $\mathbf{1 3}$ as yellow crystals in $85 \%$ yield; mp $200-202{ }^{\circ} \mathrm{C}$; IR $(\mathrm{KBr}) \vee / \mathrm{cm}^{-1} 2965,2924,2872\left(\mathrm{CH}_{\text {aliphatic }}\right), 1767$ $\left(\mathrm{C}=\mathrm{O}_{\alpha \text {-pyrone }}\right), 1666\left(\mathrm{C}=\mathrm{O}_{\text {quinolone }}\right), 1615\left(\mathrm{C}=\mathrm{C}_{\text {aromatic }}\right) ;{ }^{1} \mathrm{H} N M R$ $\left(400 \mathrm{MHz}, \mathrm{CDCl}_{3}\right) \delta 1.01\left(\mathrm{t}, J 7.5 \mathrm{~Hz}, 3 \mathrm{H}, \mathrm{C} 4{ }^{\prime}-\mathrm{Hs}\right)$, 1.47-1.51 (m, 2H, C3'-Hs), 1.73-1.75 (m, 2H, C2'-Hs), 4.29 (t, 2H, $\left.J 8 \mathrm{~Hz}, \mathrm{C} 1^{\prime}-\mathrm{Hs}\right), 7.41-7.43$ (m, 2H, C9-H and C7-H), 7.84 (dd, $1 \mathrm{H}, J 8.1,1.5 \mathrm{~Hz}, \mathrm{C} 8-\mathrm{H}), 8.26(\mathrm{~d}, 1 \mathrm{H}$, $J 8.1 \mathrm{~Hz}, \mathrm{C} 10-\mathrm{H}) ;{ }^{13} \mathrm{C}$ NMR $\left(101 \mathrm{MHz}, \mathrm{CDCl}_{3}\right) \delta 13.7$ (C4'), 20.1 (C3'), 29.5 (C2'), 42.3 (C1'), 90.7, 99.8, 113.8, 115.0, 124.0, 124.8, 129.6, 134.1, 138.1, 159.2, 162.7, 168.9; ESI-MS $m / z, 349.7[\mathrm{M}+\mathrm{H}]^{+}$and $371.7[\mathrm{M}+\mathrm{Na}]^{+}$; anal. calcd. for $\mathrm{C}_{16} \mathrm{H}_{13} \mathrm{ClN}_{2} \mathrm{O}_{5}$ (348.75): C 55.11, H 3.76, N 8.03, found: C 55.01, H 3.67, N 8.01\%.

\section{3-Amino-6-butyl-4-chloropyrano[3,2-c]quinoline- 2,5(6H)-dione (14)}

A mixture of compound $\mathbf{1 3}$ (3.48 g, $10 \mathrm{mmol})$, tin metal powder $(4.00 \mathrm{~g}, 33 \mathrm{mmol})$ and concentrated $\mathrm{HCl}$ $(30 \mathrm{~mL})$ was stirred at $130{ }^{\circ} \mathrm{C}$ for $1 \mathrm{~h}$. Methanol $(25 \mathrm{~mL})$ was added to the reaction mixture. The mixture was heated under reflux for $2 \mathrm{~h}$ until the color became clear yellow. The reaction mixture was filtered on hot and the filtrate poured on ice $(200 \mathrm{~g})$. The obtained solid was filtered, dried under vacuum and crystallized from ethanol (96\%) to produce $\mathbf{1 4}$ 
as yellow crystals in $75 \%$ yield; $\mathrm{mp} 181-183^{\circ} \mathrm{C}$; $\mathrm{IR}(\mathrm{KBr})$ $\mathrm{v} / \mathrm{cm}^{-1}$ 3526, $3380\left(\mathrm{NH}_{2}\right), 2959,2921,2848\left(\mathrm{CH}_{\text {aliphatic }}\right)$, $1722\left(\mathrm{C}=\mathrm{O}_{\alpha \text {-pyrone }}\right), 1666\left(\mathrm{C}=\mathrm{O}_{\text {quinolone }}\right), 1613(\mathrm{C}=\mathrm{C}) ;{ }^{1} \mathrm{H}$ NMR $\left(400 \mathrm{MHz}, \mathrm{DMSO}-d_{6}\right) \delta 0.92\left(\mathrm{t}, J 7.5 \mathrm{~Hz}, 3 \mathrm{H}, \mathrm{C}^{\prime} '-\mathrm{Hs}\right)$, 1.35-1.41 (m, 2H, C3'-Hs), 1.63-1.67 (m, 2H, C2'-Hs), 4.31 (t, $\left.2 \mathrm{H}, J 7.6 \mathrm{~Hz}, \mathrm{Cl}{ }^{\prime}-\mathrm{Hs}\right), 6.97$ (bs, $\left.2 \mathrm{H}, \mathrm{NH}_{2}\right), 7.43(\mathrm{dd}, 1 \mathrm{H}$, $J$ 8.0, $1.2 \mathrm{~Hz}, \mathrm{C} 9-\mathrm{H}), 7.74$ (d, 1H, J $8.0 \mathrm{~Hz}, \mathrm{C} 7-\mathrm{H}), 8.05$ (dd, $1 \mathrm{H}, J$ 8.0, $1.2 \mathrm{~Hz}, \mathrm{C} 8-\mathrm{H}), 8.13(\mathrm{~d}, 1 \mathrm{H}, J 8.0 \mathrm{~Hz}, \mathrm{C} 10-\mathrm{H})$; ${ }^{13} \mathrm{C}$ NMR (101 MHz, DMSO- $d_{6}$ ) $\delta 13.1$ (C4'), 19.9 (C3'), 29.7 (C2'), 42.1 (C1'), 101.7, 114.0, 115.0, 116.3, 122.7, 124.3, 132.1, 136.1, 146.4, 148.8, 158.9, 162.8; ESI-MS $\mathrm{m} / z, 319.2[\mathrm{M}+\mathrm{H}]^{+}$and $341.7[\mathrm{M}+\mathrm{Na}]^{+}$; anal. calcd. for $\mathrm{C}_{16} \mathrm{H}_{15} \mathrm{ClN}_{2} \mathrm{O}_{3}$ (318.76): $\mathrm{C} 60.29, \mathrm{H} 4.74, \mathrm{~N} 8.79$, found: C 60.19, H 4.66, N 8.71\%.

\section{6-Butyl-2-methyl-6H-[1,4]oxazino[2', 3':4,5]pyrano} [3,2-c]quinoline-3,5,12-trione (15)

A mixture of compound $14(3.18 \mathrm{~g}, 10 \mathrm{mmol})$ and sodium pyruvate $(1.10 \mathrm{~g}, 10 \mathrm{mmol})$, in DMF $(50 \mathrm{~mL})$ was refluxed for $8 \mathrm{~h}$. The solid deposited during heating, was isolated by filtration, air dried and crystallized from ethanol (96\%) to give the compound $\mathbf{1 5}$ as pale yellow crystals in $61 \%$ yield; $\mathrm{mp}>300^{\circ} \mathrm{C}$; IR $(\mathrm{KBr}) \mathrm{v} / \mathrm{cm}^{-1} 2953$, 2924, $2866\left(\mathrm{CH}_{\text {aliphatic }}\right), 1755(2 \mathrm{C}=\mathrm{O}), 1650\left(\mathrm{C}=\mathrm{O}_{\text {quinolone }}\right)$, $1613(\mathrm{C}=\mathrm{N}), 1586\left(\mathrm{C}=\mathrm{C}_{\text {aromatic }}\right) ;{ }^{1} \mathrm{H}$ NMR $(400 \mathrm{MHz}$, DMSO- $\left.d_{6}\right) \delta 0.94$ (t, $3 \mathrm{H}, J 7.3 \mathrm{~Hz}, \mathrm{C} 4$ '-Hs), 1.38-1.43 (m, 2H, C3'-Hs), 1.58-1.64 (m, 2H, C2'-Hs), 2.67 (s, 3H, $\mathrm{CH}_{3}$ ), 4.30 (t, $2 \mathrm{H}, J 7.8 \mathrm{~Hz}, \mathrm{C} 1$ '-Hs), 7.41 (dd, 1H, J 8.1, $1.4 \mathrm{~Hz}, \mathrm{C} 9-\mathrm{H}), 7.67-7.80$ (m, 2H, C7-H and C8-H), 8.11 $(\mathrm{d}, 1 \mathrm{H}, J 8.1 \mathrm{~Hz}, \mathrm{C} 10-\mathrm{H}) ;{ }^{13} \mathrm{C}$ NMR $\left(101 \mathrm{MHz}\right.$, DMSO- $\left.d_{6}\right)$ $\delta 13.0\left(\mathrm{C}^{\prime}\right), 14.3\left(\mathrm{CH}_{3}\right), 19.9\left(\mathrm{C}^{\prime}\right), 37.9\left(\mathrm{C}^{\prime}\right), 41.5\left(\mathrm{Cl}^{\prime}\right)$, 96.2, 99.7, 113.5, 116.6, 124.4, 124.8, 135.2, 138.2, 142.9, 158.5, 159.2, 162.2, 163.0, 167.6; ESI-MS $\mathrm{m} / \mathrm{z}, 353.5$ $[\mathrm{M}+\mathrm{H}]^{+}$and $375.4[\mathrm{M}+\mathrm{Na}]^{+}$; anal. calcd. for $\mathrm{C}_{19} \mathrm{H}_{16} \mathrm{~N}_{2} \mathrm{O}_{5}$ (352.35): C 64.77, H 4.58, N 7.95, found: C 64.71, H 4.55, N 7.90\%.

\section{4-Butyl-7,14-dihydro-benzo[1,4]oxazino[2',3':4,5]pyrano} [3,2-c]quinoline-6,13-dione (16)

To a solution of compound $\mathbf{1 4}(3.18 \mathrm{~g}, 10 \mathrm{mmol})$ in DMF $(50 \mathrm{~mL})$, were added 2-bromophenol $(1.15 \mathrm{~mL}$, $10 \mathrm{mmol})$ and $\mathrm{NaH}(0.48 \mathrm{~g}, 20 \mathrm{mmol})$. The mixture was refluxed for $12 \mathrm{~h}$, at which point TLC analysis indicated that the reaction was completed. The reaction mixture was then poured into ice cold water $(100 \mathrm{~mL})$. The precipitate was collected by filtration and washed with water, $\mathrm{EtOH}$, and ether. Recrystallization from $\mathrm{AcOH}$ gave pure $\mathbf{1 6}$ as yellow crystals in $60 \%$ yield; $\mathrm{mp}>300^{\circ} \mathrm{C}$; $\mathrm{IR}(\mathrm{KBr}) \mathrm{v} / \mathrm{cm}^{-1}$ $3356(\mathrm{NH}), 2954,2930,2864\left(\mathrm{CH}_{\text {aliphatic }}\right), 1710\left(\mathrm{C}=\mathrm{O}_{\alpha-\text { pyrone }}\right)$, $1677\left(\mathrm{C}=\mathrm{O}_{\text {quinolone }}\right), 1615\left(\mathrm{C}=\mathrm{C}_{\text {aromatic }}\right) ;{ }^{1} \mathrm{H} \mathrm{NMR}(400 \mathrm{MHz}$, DMSO- $\left.d_{6}\right) \delta 0.99$ (t, 3H, $\left.J 7.5 \mathrm{~Hz}, \mathrm{C}^{\prime}{ }^{\prime}-\mathrm{Hs}\right), 1.46-1.52$ (m, 2H, C3'-Hs), 1.75-1.81 (m, 2H, C2'-Hs), 4.44 (t, 2H, $J 7.6 \mathrm{~Hz}, \mathrm{C} 1$ '-Hs), 7.13 (d, 1H, J 6.6 Hz, $\left.\mathrm{H}_{\text {benzo }}\right), 7.34$ (dd, $1 \mathrm{H}, J$ 8.1, $1.1 \mathrm{~Hz}, \mathrm{C} 3-\mathrm{H}), 7.46-7.52(\mathrm{~m}, 2 \mathrm{H}, \mathrm{C} 1-\mathrm{H}$ and $\left.\mathrm{H}_{\text {benzo }}\right)$, 7.76-8.12 (m, $3 \mathrm{H}, \mathrm{C} 2-\mathrm{H}$ and $\left.2 \mathrm{H}_{\text {benzo }}\right), 8.23(\mathrm{~d}, 1 \mathrm{H}$, $J 8.1 \mathrm{~Hz}, \mathrm{C} 4-\mathrm{H}), 9.12(\mathrm{~s}, 1 \mathrm{H}, \mathrm{NH}) ;{ }^{13} \mathrm{C}$ NMR $(101 \mathrm{MHz}$, DMSO- $d_{6}$ ) $\delta 14.1$ (C4'), 19.9 (C3'), 31.1 (C2'), 42.2 (C1'), $100.8,112.4,113.7,114.0,115.0,116.3,122.7,123.2$, $124.3,132.2,133.3,136.9,144.4,151.2,152.1,159.2$, 162.8, 163.0; ESI-MS $\mathrm{m} / \mathrm{z}, 375.1[\mathrm{M}+\mathrm{H}]^{+}$and 397.3 $[\mathrm{M}+\mathrm{Na}]^{+}$; anal. calcd. for $\mathrm{C}_{22} \mathrm{H}_{18} \mathrm{~N}_{2} \mathrm{O}_{4}(374.40)$ : C 70.58, H 4.85, N 7.48, found: C 70.50, H 4.78, N 7.43\%.

\section{Results and Discussion}

The 3-amino-4-hydroxypyranoquinolinone (1), as 1,4-bifunctional nucleophiles, represents a good building block for synthesis of many analogues of 1,4-oxazinopyranoquinolinediones, via its heterocyclization reactions with a variety of 1,2-dichloro derivatives. Thus, cyclocondensation of compound $\mathbf{1}$ with oxalyl chloride in dry dichloromethane gave oxazinopyranoquinolinone $\mathbf{2}$, which was present as interconverting keto and enol tautomers (Scheme 1). The IR spectrum of the product exhibited absorption bands at 1744, 1703, 1662 and $1633 \mathrm{~cm}^{-1}$ attributed to the four $\mathrm{C}=\mathrm{O}$ groups of pyrone, 2,3-oxazindione and quinolinone, respectively. The ${ }^{1} \mathrm{H}$ NMR spectrum displayed evidences for existence of keto and enol form of compound 2 . This was noticed through the duplication of benzo protons and $\mathrm{NH} / \mathrm{OH}$ proton values. The ESI-MS of compound $\mathbf{2}$ showed two intense peaks at $m / z, 355.1$ and 377.1 corresponding to $[\mathrm{M}+\mathrm{H}]^{+}$and $[\mathrm{M}+\mathrm{Na}]^{+}$, respectively.

In order to obtain a novel series of 1,4-oxazinopyranoquinolinone, the reaction of the amine $\mathbf{1}$ was studied towards some ortho-dichloroheterocycles, as 1,2-bifunctional electrophiles. Thus, condensation of compound 1 with 2,3-dichloropyridine in stirring THF, at room temperature, may lead to the $N$-arylated adduct $\mathbf{3}$ or the $o$-arylated adduct 4. Analytical and spectral results for the product of this reaction evoked the proposal of obtaining 6-butyl-3-(3-chloro-pyridin-2-ylamino)-4-hydroxypyrano $[3,2-c]$ quinolone-2,5(6H)dione (3) (Scheme 1). The formation of compound $\mathbf{3}$ is happening as a result of easy nucleophilic attack of the amino group at 4-position of pyran ring to the chloride at 2-position in 2,3-dichloropyridine which is more suitable for nucleophilic substitution than that at 3-position. IR spectrum indicated that the product did not posses amino group which participates in a nucleophilic substitution reaction. IR spectrum showed two characteristic vibrational absorption bands at $v_{\max }=3458$ and $3220 \mathrm{~cm}^{-1}$ which corresponded to $\mathrm{OH}$ and 
NH functionalities. The ${ }^{1} \mathrm{H}$ NMR spectrum of compound $\mathbf{3}$ showed two deuterium-exchangeable singlet signals at 9.27 and 13.98 ppm assignable to $(\mathrm{NH})$ and $(\mathrm{OH})$ protons, respectively. Intermolecular heterocyclization at face $c$ of the pyran moiety can take place when compound $\mathbf{3}$ is heated with DMF containing $\mathrm{NaH}$. Since, the chlorine atom at position-3 of pyridine ring is susceptible to nucleophilic substitution by the $\mathrm{OH}$ function at position- 4 of pyran moiety to afford polyfused heterocyclic system 5 (Scheme 1). Evidence for the formation of 1,4-oxazinopyrano[3,2-c] quinolinedione derivative (5) is from its IR and ${ }^{1} \mathrm{H}$ NMR spectra which showed the absence of the hydroxyl group. Furthermore, the ESI-MS spectrum of compound 5 exhibited its $[\mathrm{M}+\mathrm{Na}]^{+}$ion $(\mathrm{m} / \mathrm{z}$ 398.1), which is in accordance with its structure. In order to obtain another derivative of 16-butyl-7,16-dihydro-quinoxalino $\left[2^{\prime \prime}, 3^{\prime \prime}: 5^{\prime}, 6^{\prime}\right]-1,4$-oxazino [2',3':4,5]pyrano[3,2-c] quinoline-6,15-dione (6), we have studied the reaction of compound 1 with 2,3-dichloroquinoxaline ${ }^{21}$ in boiling DMF containing NaH. IR spectrum of product 6 showed the absence of two stretching vibration bands due to the amino group, and the appearance of a new stretching signal due to $\mathrm{N}-\mathrm{H}$ group at $3292 \mathrm{~cm}^{-1}$. The $\mathrm{N}-\mathrm{H}$ proton is observed at $9.01 \mathrm{ppm}$ as a singlet signal in the ${ }^{1} \mathrm{H}$ NMR spectrum of compound $\mathbf{6}$. Furthermore, compound $\mathbf{6}$ showed a quasi-molecular ion peak at $\mathrm{m} / z 427.5[\mathrm{M}+\mathrm{H}]^{+}$and a sodiated molecular ion peak at $\mathrm{m} / z 449.4[\mathrm{M}+\mathrm{Na}]^{+}$in the positive ESI-MS.

Next, compound $\mathbf{1}$ was used for the synthesis of a novel tetra-fused system, 6-butyl-3-phenyl[1,4]oxazino $\left[2^{\prime}, 3^{\prime}: 4,5\right]$ pyrano [3,2-c] quinoline-5,12(6H)-dione (7), via the cyclo condensation with phenacyl bromide in boiling DMF containing $\mathrm{NaH}$ (Scheme 2). The ${ }^{1} \mathrm{H}$ NMR spectrum of compound $\mathbf{7}$ showed a characteristic singlet at $7.29 \mathrm{ppm}$ attributed to the oxazine $\mathrm{CH}$, in addition to an exchangeable signal at $8.94 \mathrm{ppm}$ assigned to the $\mathrm{NH}$ proton. ${ }^{13} \mathrm{C}$ NMR of compound 7 represents twenty separated signals in the region 97.2-162.7 ppm belonging to the aromatic carbon atoms of tetracyclic system and phenyl group. ESI-MS spectrum of compound 7 showed the $[\mathrm{M}+\mathrm{H}]^{+}$ion at $m / z 401.5$ and the $[\mathrm{M}+2 \mathrm{H}]^{2+}$ ion at 402.2 , the sodium adduct $[\mathrm{M}+\mathrm{Na}]^{+}$ion is also observed at $m / z$ 423.3. Similarly, cyclocondensation of compound $\mathbf{1}$ with chloroacetic acid under the same reaction conditions produced 26-butyl-3-hydroxy [1,4] oxazino[2',3':4,5]pyrano [3,2-c]quinoline-5,12(6H)-dione (9) in low yield (48\%) (Scheme 2). The ${ }^{1} \mathrm{H}$ NMR spectrum of product 9 suggests that it exists as interconverting keto and enol tautomers in deuterium dimethylsulfoxide solution. The ${ }^{1} \mathrm{H}$ NMR spectrum of compound 9 showed a new characteristic singlet signal at $7.33 \mathrm{ppm}$ characteristic of the oxazine $\mathrm{CH} .{ }^{13} \mathrm{C}$ NMR spectrum of compound 9 demonstrates the presence of fourteen $\mathrm{sp}^{2}$ hybrid carbons in region 113.4$175.5 \mathrm{ppm}$ due to the aromatic tetracyclic system. As well as, the result of the ESI-MS $[\mathrm{M}+\mathrm{H}]^{+}$ion peak at $(\mathrm{m} / \mathrm{z}, 341.3$, $100 \%$ ) is in accordance with its structure. Our attempts to improve the yield of the above reaction by changing the reaction conditions were not successful.

We expected that an alternative method for the preparation of compound $\mathbf{9}$ through a two step reaction could produce better results. Thus, the $N$-alkylated adduct $\mathbf{8}$ was prepared by treating compound $\mathbf{1}$ with bromoacetonitrile, in molar ratio $(1: 1, \mathrm{~m}: \mathrm{v})$ in boiling acetonitrile containing sodium hydride as a basic catalyst and isolated by dry chromatography using ethyl acetate/hexane 6:4 (v:v) as the eluent. The IR spectrum of the product revealed the absence of the amino group and exhibited two new stretching vibration bands at 3094 and $1744 \mathrm{~cm}^{-1}$ due to
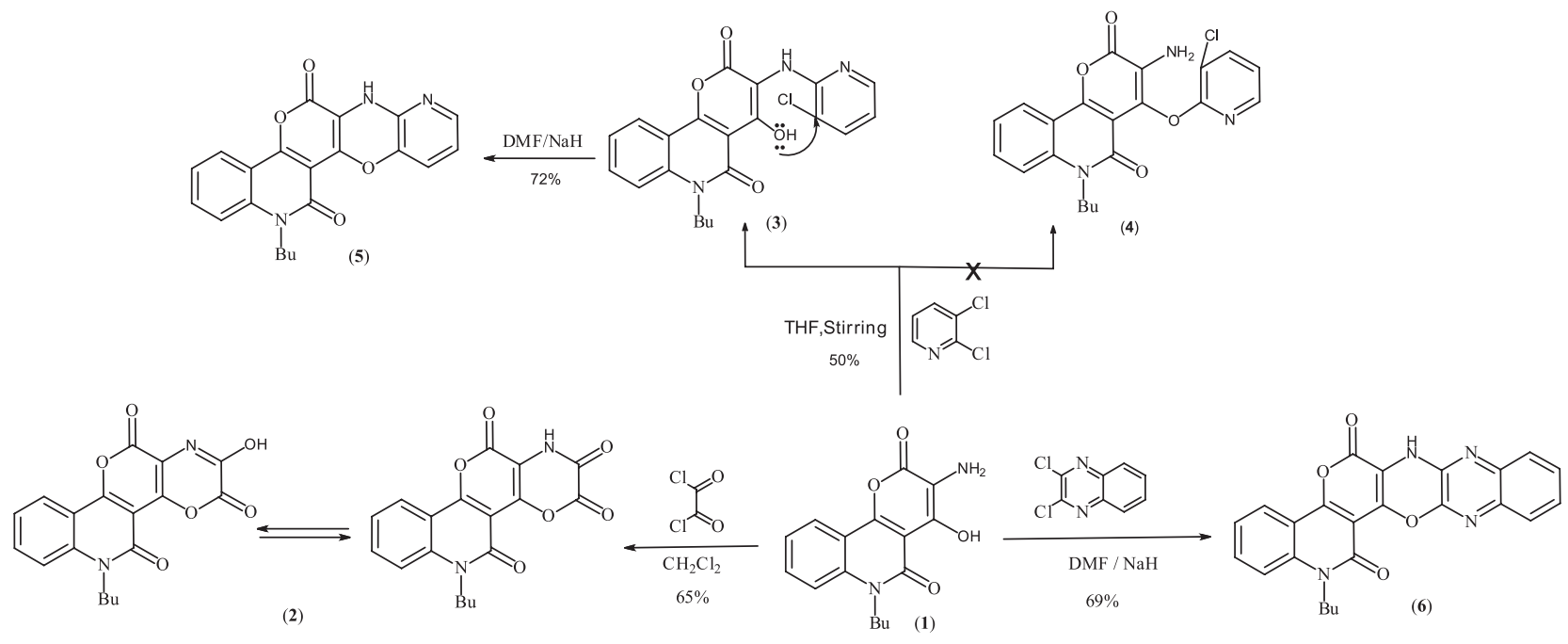

Scheme 1. Synthesis of the compounds 2-6. 


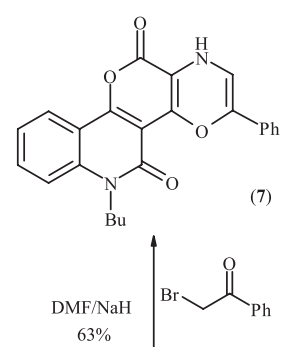

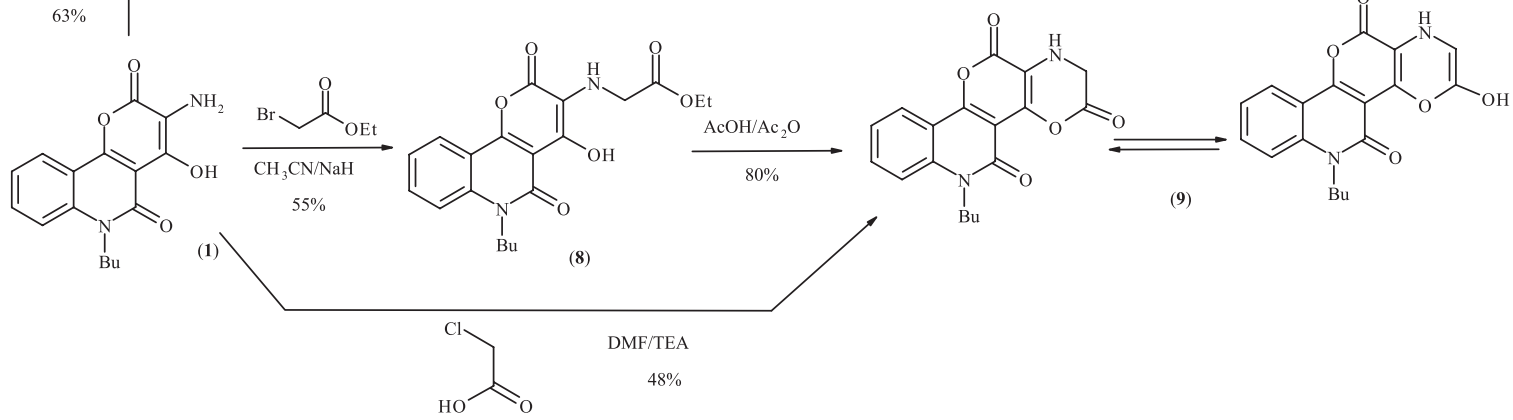

Scheme 2. Synthesis of the compounds 7-9.

$\mathrm{NH}$ and the ester $\mathrm{C}=\mathrm{O}$ groups, respectively. The ${ }^{1} \mathrm{H}$ NMR spectrum of the compound $\mathbf{8}$ confirmed the disappearance of the $\mathrm{NH}_{2}$ signal, in addition to the appearance of characteristic singlet signal at 5.23 and $6.16 \mathrm{ppm}$ assigned to the acetonitrile $\mathrm{CH}_{2}$ and $\mathrm{NH}$ group, respectively. ${ }^{13} \mathrm{C}$ NMR spectrum showed the presence of three chemical shifts at 19.9, 42.3 and $206.9 \mathrm{ppm}$ specific for ethyl group set of carbons $\mathrm{CH}_{3} \mathrm{CH}_{2} \mathrm{CO}_{2}$, besides chemical shift due to $\mathrm{CH}_{2}$ of side chain at $40.4 \mathrm{ppm}$. Boiling the $\mathrm{N}$-alkylated adduct 8 , in $\mathrm{AcOH}$ and acetic anhydride $\left(\mathrm{Ac}_{2} \mathrm{O}\right)$ affected its intramolecular heterocyclization to afford oxazine derivative 9 in good yield $80 \%$ (Scheme 2).

Continuing the synthesis of new 1,4-oxazinopyrano $[3,2-c]$ quinolone derivatives, we planned to prepare the $o$-alkylated adduct $\mathbf{1 1}$ as a synthon of further functionalized heteroannulated pyranoquinolone derivative. To approach this target, we carried out the $o$-alkylation reaction of nitropyrane $\mathbf{1 0}$ in the presence of $\mathrm{NaH}$ in acetonitrile under refluxing conditions. The $o$-alkylated adduct 11 was obtained in $47 \%$ yield. IR spectrum of compound $\mathbf{1 1}$ indicated a characteristic vibrational absorption band at $v_{\max } 2201 \mathrm{~cm}^{-1}$, specific for nitrile function. The methylene protons of acetonitrile group were observed at $4.21 \mathrm{ppm}$ in the ${ }^{1} \mathrm{H}$ NMR spectrum, while the $\mathrm{sp}^{3}$ hybridized carbon atom of the active methylene group appeared at $56.4 \mathrm{ppm}$ in the ${ }^{13} \mathrm{C}$ NMR spectrum. ESI-MS spectrum of compound $\mathbf{1 1}$ revealed quasi-molecular ion peak at $m / z 370.4[\mathrm{M}+\mathrm{H}]^{+}$and a sodiated molecular ion peak at $m / z 392.4[\mathrm{M}+\mathrm{Na}]^{+}$.

In the second step, we were mainly interested in the reduction of the nitro functionality followed by cyclization to afford the corresponding 1,4-benzoxazine (12). Encouraged by earlier results with Fe/acetic acid system for double reductive cyclization reactions, ${ }^{22,23}$ we examined the reduction of adduct $\mathbf{1 1}$ in the presence of Fe/acetic acid under refluxing conditions. We were pleased to obtain 1,4-oxazinopyranoquinolinone derivative (12) in $59 \%$ yield. The IR spectrum of compound $\mathbf{1 2}$ confirmed the absence of the nitrile group. While the ${ }^{1} \mathrm{H}$ NMR spectrum of compound $\mathbf{1 2}$ was characterized by the presence of a singlet signal at $8.98 \mathrm{ppm}$ corresponding to the protonofoxazine moiety, in addition to two deuterium exchangeable signals at $\delta 5.58$ and $13.22 \mathrm{ppm}$ due to $\mathrm{NH}$ and $\mathrm{OH}$ protons. The ESI-MS analysis of compound $\mathbf{1 2}$ showed $[\mathrm{M}+\mathrm{H}]^{+}$ion at $\mathrm{m} / \mathrm{z} 341.4$, the abundant $[\mathrm{M}+\mathrm{Na}]^{+}$ion is also observed at $\mathrm{m} / \mathrm{z}$ 363.3. The same compound was authentically obtained from the reaction of the amine $\mathbf{1}$ with chloroacetyl chloride in moderate yield $61 \%$, thus confirming its structure as that of $\mathbf{1 2}$ (Scheme 3).

Chlorination of compound $\mathbf{1 0}$ with phosphoryl chloride in the presence of triethylamine afforded 6-butyl-4-chloro3-nitropyrano[3,2-c] quinoline-2,5(6H)-dione (13) (Scheme 4). The disappearance of hydroxyl group is the prominent feature in the IR and ${ }^{1} \mathrm{H} N M R$ spectra of compound 13. ESI-MS spectrum of compound $\mathbf{1 3}$ showed $[\mathrm{M}+\mathrm{H}]^{+}$ion at $\mathrm{m} / \mathrm{z} 349.7$ and $[\mathrm{M}+\mathrm{Na}]^{+}$ion at 371.7. The $[2 \mathrm{M}+\mathrm{Na}]^{+}$ ion is also observed at $\mathrm{m} / \mathrm{z}$ 719.7. The nitro derivative (13) was reduced under stannous-catalyzed condition to produce a novel 3-amino-6-butyl-4-chloropyrano[3,2-c]quinoline2,5(6H)-dione (14) in 75\% yield (Scheme 4). The formation of the amino analogue was confirmed from its FTIR spectrum, which showed stretching vibrational bands at 3526 and $3380 \mathrm{~cm}^{-1}$ as a double peak due to the $\mathrm{NH}_{2}$ group. The ${ }^{1} \mathrm{H}$ NMR spectrum of compound $\mathbf{1 4}$ showed deuterium exchangeable singlet signal at $6.97 \mathrm{ppm}$ characteristic of an $\mathrm{NH}_{2}$ group. Compound 14 showed a quasi-molecular ion peak 


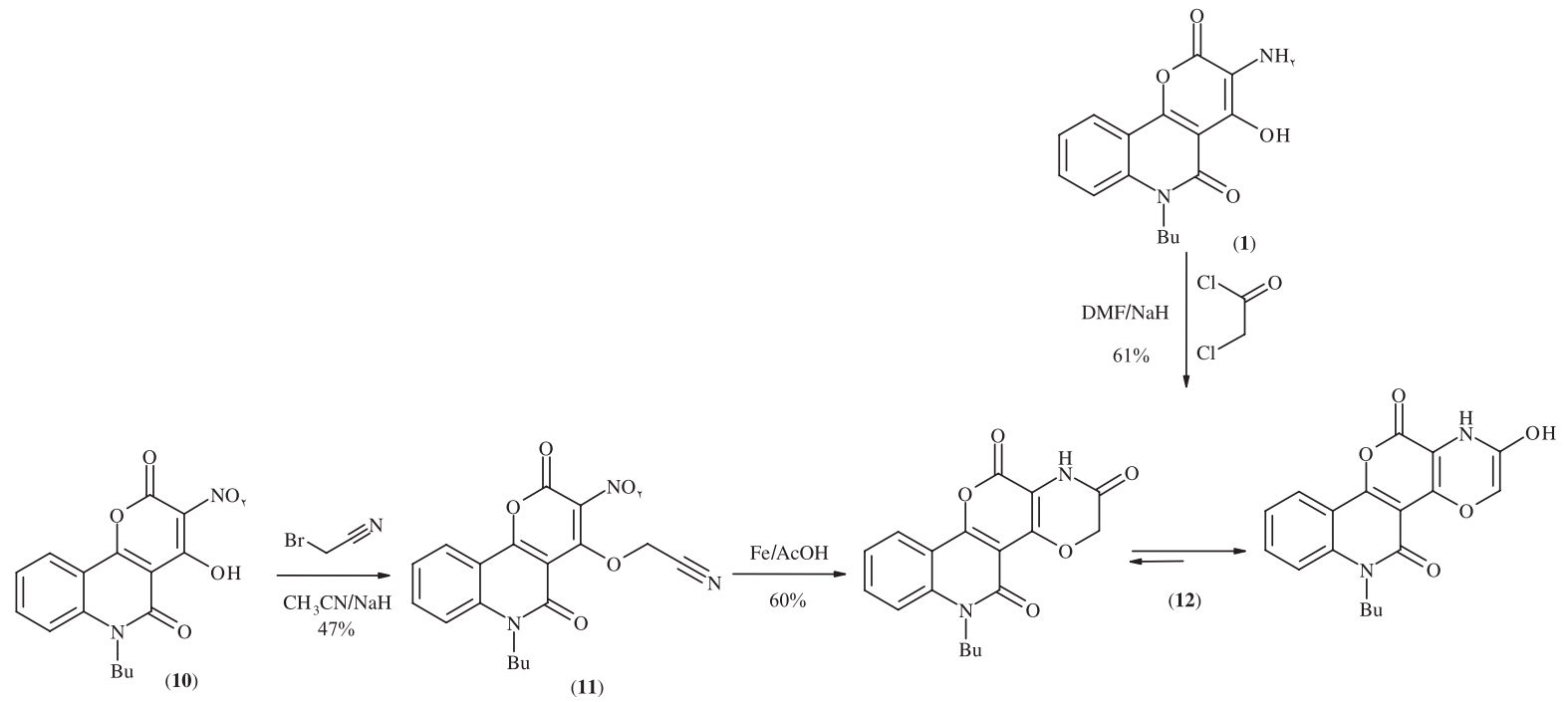

Scheme 3. Synthesis of the compounds 10-12.

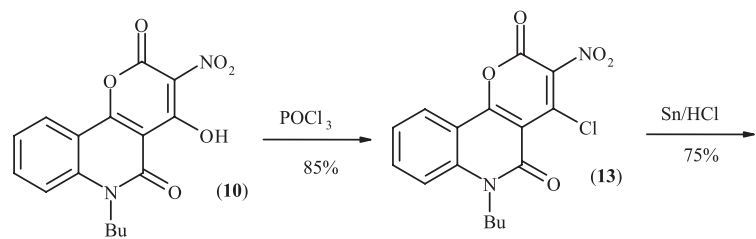<smiles>CC(=O)C(=O)Nc1c(Cl)c2c(=O)n(C)c3ccccc3c2oc1=O</smiles><smiles>Cc1nc2c(=O)oc3c(c2oc1=O)-c1ccccc1C(C)N3C</smiles><smiles>CC(C)[C@H](C)Oc1ccccc1Br</smiles><smiles>Cn1c(=O)c2c3ccccc3oc2c2c3ccccc3[nH]c21</smiles>

Scheme 4. Synthesis of the compounds 13-16.

at $m / z 319.2[\mathrm{M}+\mathrm{H}]^{+}$and a sodiated molecular ion peak at $\mathrm{m} / \mathrm{z} 341.7[\mathrm{M}+\mathrm{Na}]^{+}$in the positive ESI-MS corresponding to $\mathrm{C}_{16} \mathrm{H}_{15} \mathrm{ClN}_{2} \mathrm{O}_{3}$ formula. Another novel tetraheterocyclic system, 1,4-oxazino pyranoquinolinone (15) was afforded when the compound $\mathbf{1 4}$ was treated with sodium pyruvate. The IR spectrum of the product exhibited three characteristic absorption bands at 1755 and $1650 \mathrm{~cm}^{-1}$ attributed to the $\mathrm{C}=\mathrm{O}$ groups of pyrone, oxazinone and quinolone, respectively. The methyl proton signal at position 2 of the oxazine ring was observed as a single peak at $2.67 \mathrm{ppm}$ in the ${ }^{1} \mathrm{H}$ NMR spectrum, while the $\mathrm{sp}^{3}$ hybridized carbon atom of the methyl group appeared at $\delta 14.3$ in the ${ }^{13} \mathrm{C}$ NMR spectrum. The ESI-MS of compound $\mathbf{1 5}$ showed four abundant peaks at $\mathrm{m} / \mathrm{z} 353.5,354.2,375.4$ and 727.3, corresponding to $[\mathrm{M}+\mathrm{H}]^{+},[\mathrm{M}+2 \mathrm{H}]^{2+}$, $[\mathrm{M}+\mathrm{Na}]^{2+}$ and $[2 \mathrm{M}+\mathrm{Na}]^{+}$, respectively. Finally, treatment of compound $\mathbf{1 4}$ with 2-bromophenol yielded 14-butyl-7,14-dihydro-benzo[1,4]oxazino[2',3':4,5]pyrano [3,2-c]quinoline-6,13-dione (16). The ${ }^{1} \mathrm{H}$ NMR spectrum of compound $\mathbf{1 6}$ showed signals attributed to the butyl and eight aromatic protons, in addition to an exchangeable signals attributed to the $\mathrm{NH}$ protons at $9.12 \mathrm{ppm}$. In the ${ }^{13} \mathrm{C}$ NMR spectrum there are eighteen characteristic downfield signals at 100-163 ppm which are attributed to the aromatic carbon atoms of pentacyclic system. Most abundant parent ion was observed in positive ESI-MS of compound 16 as protonated molecule $[\mathrm{M}+\mathrm{H}]^{+}$with $\mathrm{m} / \mathrm{z}$ of 375.1. The simple sodium adduct ion $[\mathrm{M}+\mathrm{Na}]^{+}$was also observed at $m / z$ 397.3.

\section{Conclusions}

Combination of 1,4-oxazine nucleus with pyranoquinolinone moiety in one molecular frame was 
successfully achieved via heterocyclization reactions of 3,4-bifunctional pyranoquinolinone with different reagents.

\section{Supplementary Information}

Supplementary data (IR, mass, ${ }^{1} \mathrm{H}$ and ${ }^{13} \mathrm{C}$ NMR spectra) are available free of charge at http://jbcs.sbq.org.br as a PDF file.

\section{Acknowledgments}

The authors thank the Research School of Chemistry (RSC) at the Australian National University (ANU), Canberra, ACT 0200, Australia, for generous instrumentation support. Mohamed M. Hassan is highly grateful and indebted to Professor Martin G. Banwell, Research School of Chemistry, Australian National University, for valuable guidance, encouragement and facilities in the measurement of spectral data during the period of Mohamed M. Hassan's scientific mission at the RSC-ANU.

\section{References}

1. Schreiber, S. L.; Science 2000, 287, 1964.

2. Thompson, L. A.; Curr. Opin. Chem. Biol. 2000, 4, 324.

3. Singh, M. S.; Nandi, G. C.; Samai, S.; Green Chem. 2012, 14, 447.

4. Wen, L. R.; Li, Z. R.; Li, M.; Cao, H.; Green Chem. 2012, 14, 707.

5. Bansal, R. K.; Heterocyclic Chemistry, $4^{\text {th }}$ ed.; New Age International: New Delhi, India, 2014.

6. Turgut, Z.; Pelit, E.; Köycü, A.; Molecules 2007, 12, 345.

7. Smid, P.; Coolen, H. K. A. C.; Keizer, H. G.; Van Hes, R.; De Moes, J. P.; Den Hartog, A. P.; Stork, B.; Plekkenpol, R. H.; Niemann, L. C.; Stroomer, C. N. J.; Tulp, M. T. M.; van Stuivenberg, H. H.; McCreary, A. C.; Hesselink, M. B.; Herremans, A. H. J.; Kruse, C. G. J.; J. Med. Chem. 2005, 48, 6855.

8. Fringuelli, R.; Pietrella, D.; Schiaffella, F.; Guarraci, A.; Perito, S.; Bistoni, F.; Vecchiarelli, A.; Bioorg. Med. Chem. 2002, 10, 1681.
9. Macchiarulo, A.; Costantino, G.; Fringuelli, D.; Vecchiarelli, A.; Schiaffella, F.; Fringuelli, R.; Bioorg. Med. Chem. 2002, 10,3415 .

10. Lanni, T. B.; Greene, K. L.; Kolz, C. N.; Para, K. S.; Visnick, M.; Mobley, J. L.; Dudley, D. T.; Baginski, T. J.; Liimatta, M. B.; Bioorg. Med. Chem. Lett. 2007, 17, 756.

11. Huang, M.-Z.; Huang, K.-L.; Ren, Y.-G.; Lei, M.-X.; Huang, L.; Hou, Z.-K.; Liu, A.-P.; Qu, X.-M.; J. Agric. Food Chem. 2005, 53, 7908.

12. Scheunemann, M.; Sorger, D.; Kouznetsova, E.; Sabri, O.; Schliebs, R.; Wenzel, B.; Steinbach, J.; Tetrahedron Lett. 2007, $48,5497$.

13. Arasakumar, T.; Mathusalini, S.; Gopalan, S.; Shyamsivappan, S.; Ata, A.; Mohan, P. S.; Bioorg. Med. Chem. Lett. 2017, 27, 1538.

14. Magedov, I. V.; Manpadi, M.; Ogasawara, M. A.; Dhawan, A. S.; Rogelj, S.; Van slambrouck, S.; Steelant, W. F. A.; Evdokimov, N. M.; Uglinskii, P. Y.; Elias, E. M.; Knee, E. J.; Tongwa, P.; Antipin, M. Y.; Kornienko, A.; J. Med. Chem. 2008, 51, 2561.

15. Nakashima, K.-i.; Oyama, M.; Ito, T.; Akao, Y.; Witono, J. R.; Darnaedi, D.; Tanaka, T.; Murata, J.; Iinuma, M.; Tetrahedron 2012, 68, 2421.

16. Tomassoli, I.; Herlem, G.; Picaud, F.; Benchekroun, M.; Bautista-Aguilera, O. M.; Luzet, V.; Jimeno, M.-L.; Gharbi, T.; Refouvelet, B.; Ismaili, L.; Monatsh. Chem. 2016, 147, 1069.

17. Ramesh, E.; Manian, R. S.; Raghunathan, R.; Sainath, S.; Raghunathan, M.; Bioorg. Med. Chem. 2009, 17, 660.

18. Anniyappan, M.; Muralidhran, D.; Perumal, P. T.; Tetrahedron Lett. 2003, 44, 3653.

19. El-Agrody, A. M.; Abd-Rabboh, H. S. M.; Al-Ghamd, A. M.; Med. Chem. Res. 2013, 22, 1339.

20. Hassanin, H. M.; Abdou, I. M.; Saeed, A. M.; Arkivoc 2017, v, 172.

21. Romer, D. R.; J. Heterocycl. Chem. 2009, 46, 317.

22. Ramesh, C.; Raju, B. R.; Kavala, V.; Kuo, C.-W.; Yao, C.-F.; Tetrahedron 2011, 67, 1187.

23. Parvatkar, P. T.; Parameswaran, P. S.; Tilve, S. G.; Tetrahedron Lett. 2007, 48, 7870.

Submitted: September 8, 2017 Published online: November 14, 2017 